Pacific Journal of Mathematics

THE DILATATION OF SOME STANDARD MAPPINGS 


\section{THE DILATATION OF SOME STANDARD MAPPINGS}

\section{TILlA KLOTZ}

It is not unusual to consider on a surface a conformal structure determined by a positive definite quadratic form which may or may not be the official Riemannian metric on the surface. Given a smooth mapping with positive Jacobian between a pair of surfaces each provided with such a conformal structure, we describe in this paper an obvious procedure for computing the dilatation of the mapping. Next, we consider surfaces smoothly immersed in $E^{3}$, and mappings (called allowable) for which dilatation is a function of the principal curvatures at corresponding points. Referring to a conformal structure as geometrically significant if determined by a linear combination of the fundamental forms with coefficients which are smooth functions of the principal curvatures, we show (for example) that a mapping which preserves lines of curvature is allowable between any pair of geometrically significant conformal structures if it is allowable between any one pair of geometrically significant conformal structures. Finally, we prove that a complete surface smoothly immersed in $E^{3}$ on which $K \leqq 0$ and $H^{2}-K \equiv c \neq 0$ is conformally equivalent either to the finite plane or to the once punctured finite plane.

1. Computing dilatation. The material in this section is not in any sense original. (See, for example, [2], p. 103 and 118, a source suggested by the referee.) What we present is the procedure by which dilatation will be computed throughout the remainder of the paper.

Consider a pair of abstract, smooth, oriented surfaces $S$ and $\hat{S}$, and a smooth mapping

$$
f: S \rightarrow \hat{S}
$$

with positive Jacobian. Next, suppose that an arbitrary pair of smooth positive definite quadratic forms $\Lambda$ and $\hat{A}$ are used to determine conformal structures on $S$ and $\widehat{S}$, respectively, yielding Riemann surfaces $R_{A}$ and $\hat{R}_{\hat{\Lambda}}$ ([1], p. 26). At each point $p$ of $S$, the induced mapping

$$
f: R_{i} \rightarrow \hat{R}_{\hat{\Lambda}}
$$

has the dilatation $([1]$, p. 1)

$$
\left.\mathscr{K}_{f}(\Lambda, \hat{\Lambda})\right|_{p}=\frac{\max \sqrt{\frac{\left.\hat{\Lambda}\right|_{f(p)}}{\left.\Lambda\right|_{p}}}}{\min \sqrt{\frac{\left.\hat{\Lambda}\right|_{f(p)}}{\left.\Lambda\right|_{p}}}}
$$


where, for the moment, we use $\Lambda$ and $\hat{\Lambda}$ as metric tensors, comparing, as indicated on the right hand side of (1), the lengths they assign to corresponding nonzero tangent vectors at $p$ and $f(p)$ respectively. For convenience, we square both sides of (1) to obtain

$$
\left.\mathscr{K}_{f}^{2}(\Lambda, \hat{\Lambda})\right|_{p}=\frac{\max \frac{\left.\hat{\Lambda}\right|_{f(p)}}{\left.\Lambda\right|_{p}}}{\min \frac{\left.\hat{\Lambda}\right|_{f(p)}}{\left.\Lambda\right|_{p}}} .
$$

It is, however, always possible (since $f$ is locally a diffeomorphism) to express $\Lambda$ and $\hat{\Lambda}$ in terms of arbitrary local coordinates $x, y$ on $S$ throughout their domain of definition on $S$ as follows

$$
\begin{aligned}
& \Lambda=\alpha d x^{2}+2 \beta d x d y+\gamma d y^{2} \\
& \hat{\Lambda}=\widehat{\alpha} d x^{2}+2 \widehat{\beta} d x d y+\hat{\gamma} d y^{2} .
\end{aligned}
$$

The expression for dilatation in terms of the six coordinate functions $\alpha, \beta, \gamma, \hat{\alpha}, \hat{\beta}$, and $\hat{\gamma}$ is given by observing that

$$
\left.\mathscr{K}_{f}^{2}(\Lambda, \hat{\Lambda})\right|_{p}=\frac{H_{f}+\sqrt{H_{f}^{2}-K_{f}}}{H_{f}-\sqrt{H_{f}^{2}-K_{f}}},
$$

where

$$
H_{f}=\frac{\hat{\alpha} \gamma+\alpha \hat{\gamma}-2 \beta \hat{\beta}}{2\left(\alpha \gamma-\beta^{2}\right)}
$$

and

$$
K_{f}=\frac{\hat{\alpha} \hat{\gamma}-\widehat{\beta}^{2}}{\alpha \gamma-\beta^{2}}
$$

The functions $H_{f}=H_{f}(\Lambda, \hat{\Lambda})$ and $K_{f}=K_{f}(\Lambda, \hat{\Lambda})$ are, of course, independent of the choice of coordinates $x, y$.

The notation above was suggested by considering the following special situation. Take for $S$ and $\widehat{S}$ one and the same surface smoothly immersed in 3-space. Assume that Gaussian curvature $K$ is positive, and orient $S$ so that its mean curvature $H$ is positive as well. Now set $\Lambda=I, \hat{\Lambda}=I I$, and take for $f$ the identity mapping

$$
\text { id: } S \rightarrow S \text {. }
$$

Here, computation of

$$
\mathscr{K}_{i d}^{2}(I, I I)
$$

involves, by (2), taking the ratio of the greater to the lesser principal 
curvature on $S$. In this special case, $H_{f}=H$ and $K_{f}=K$. Conformal points for $f$ are characterized as umbilics on $S$.

The old formulas ([8], p. 83) giving ordinary principal curvatures in terms of the six coefficient functions of the first and second fundamental forms are exactly the formulas which still apply to compute numerator and denominator on the right hand side of (2). Thus, using (3), we obtained (4). By similar imitation ([8], p. 80), we find the directions of greatest and least stretching by $f$ (given $\Lambda$ and $\hat{\Lambda}$ ) as solutions of the equation

$$
\left|\begin{array}{ccc}
d y^{2} & -d x d y & d x^{2} \\
\alpha & \beta & \gamma \\
\widehat{\alpha} & \widehat{\beta} & \hat{\gamma}
\end{array}\right|=0 .
$$

Analogous to the lines of curvature in ordinary surface theory, we have here the integral curves of (5). These form a net with singularities only where

$$
f: R_{\Lambda} \rightarrow \hat{R}_{\hat{\Lambda}}
$$

is conformal. This special $(f, \Lambda, \hat{\Lambda})$ net can be characterized, except where $\Lambda \alpha \hat{\Lambda}$, as the only net on $S$ orthogonal in the $\Lambda$ metric which is carried by $f$ (at least locally) to a net on $\hat{S}$ orthogonal in the $\hat{\Lambda}$ metric. Corresponding to lines-of-curvature coordinates in the familiar case, we have those special $(f, A, \hat{\Lambda})$ coordinates $x, y$ in terms of which in (3)

$$
\beta \equiv \widehat{\beta} \equiv 0 .
$$

When using such special $(f, \Lambda, \hat{\Lambda})$ coordinates, dilatation has the particularly simple expression

$$
\mathscr{K}_{f}^{2}(A, \hat{\Lambda})=\max \left(\frac{\frac{\hat{\alpha}}{\hat{\alpha}}}{\frac{\hat{\gamma}}{\gamma}}, \frac{\hat{\gamma}}{\frac{\hat{\alpha}}{\alpha}}\right) .
$$

2. Allowable mappings. Henceforth, we assume that the oriented surfaces $S$ and $\widehat{S}$ are smoothly immersed in $E^{3}$. Among the various structures $R_{A}$ available on an $S$ in $E^{3}$ are two already noted, ordinary $R_{I}$ structure, and the structure $R_{I I}$ ([5]) defined wherever $H$ and $K$ are positive. Three other structures $R_{I I^{\prime}}, R_{I I I}$ and $R_{I+I I I}$ will be specifically alluded to below. The first $R_{I I^{\prime}}$ is defined wherever $K<0$ on $S$ by the form

$$
I I^{\prime}=\sqrt{H^{2}-K}(H I I-K I)
$$


([6]). The second $R_{I I I}$ is determined wherever $K \neq 0$ on $S$ by the form

$$
I I I=2 H I I-K I,
$$

and eliminates need for explicit reference to the unit sphere when only conformal properties of the spherical image mapping are of interest. Finally, the third structure $R_{I+I I I}$ is cited as one example of a simple, nonstandard conformal structure globally definable on an $S$ in $E^{3}$ without restrictions on curvature. The following definition is meant to characterize those $\Lambda$ which can be expected to yield interesting structures of the sort just cited.

Definition. A positive definite $\Lambda$ on a smoothly immersed surface in $E^{3}$ is geometrically significant if and only if

$$
\Lambda=g_{I}\left(k_{1}, k_{2}\right) I+g_{I I}\left(k_{1}, k_{2}\right) I I
$$

where the smooth, real valued functions $g_{I}$ and $g_{I I}$ depend only upon the values of principal curvatures $k_{1}$ and $k_{2}$ on $S$. We call $R_{A}$ geometrically significant if and only if $\Lambda$ is.

The sort of mappings $f: R_{\Lambda} \rightarrow \hat{R}_{\hat{\Lambda}}$ which will concern us here are those (still with positive Jacobian) for which standard restrictions such as quasi-conformality have immediate interpretations in terms of geometric quantities on $S$ and $\widehat{S}$. More specifically, consider the class of mappings defined below.

Definition. $f: R_{A} \rightarrow \hat{R}_{\hat{\Lambda}}$ is allowable if and only if the dilatation

$$
\mathscr{K}_{f}(\Lambda, \hat{\Lambda})=g\left(k_{1}, k_{2} ; \hat{k}_{1}, \hat{k}_{2}\right)
$$

can be expressed as a function of the four principal curvature values at points of $S$ and $\widehat{S}$ in correspondence under $f$.

Note that $\Lambda$ and $\hat{\Lambda}$ need not be geometrically significant for $f$ to be allowable. Any $f: R_{A} \rightarrow \hat{R}_{\hat{\Lambda}}$ with constant dilatation is automatically allowable. We shall be concerned however with $f$ 's which are allowable between geometrically significant conformal structures, and as an aid in their study, we prove the following result.

Lemma. Let $f: S \rightarrow \widehat{S}$ preserve lines of curvature. Then if $f$ is allowable for one pair of geometrically significant forms $\Lambda$ and $\hat{\Lambda}, f$ is allowable for all pairs of geometrically significant forms $\Lambda$ and $\hat{\Lambda}$. 
Proof. Away from irremovable umbilics on $S$, introduce, locally, lines-of-curvature coordinates $x, y$. Then, since $f$ preserves lines of curvature, we have for any geometrically significant pair of forms $\Lambda, \hat{\Lambda}$ that

$$
\begin{aligned}
& \left.{ }^{(\hat{I})}=\stackrel{(}{\mathrm{E}} d x^{2}+{ }^{(} \hat{G}\right) d y^{2},
\end{aligned}
$$

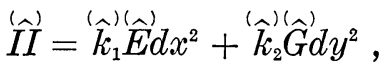

and

$$
\hat{\Lambda}=(\hat{g})+\left(\hat{k}_{1}\right)\left(\hat{g_{I I}}\right)(\hat{E}) d x^{2}+\left(\hat{g_{I}}+\hat{k}_{2} \hat{g}_{I I}\right)(\hat{G}) d y^{2} .
$$

Thus, by (6),

$$
\mathscr{K}_{f}^{2}(\Lambda, \hat{\Lambda})=\max \left\{\frac{\frac{\left(\hat{g}_{I}+\hat{k}_{1} \hat{g}_{I I}\right) \hat{E}}{\left(\frac{\left.g_{I}+k_{1} g_{I I}\right) E}{\left(\hat{g}_{I}+\hat{k}_{2} \hat{g}_{I I}\right) \hat{G}}\right.}, \frac{\left(\hat{g}_{I}+\hat{k}_{2} \hat{g}_{I I}\right) \hat{G}}{\left(g_{I}+k_{2} g_{I I}\right) G} \frac{\left(\hat{g}_{I}+\hat{k}_{1} g_{I I}\right) G}{\left(g_{I}+\hat{g}_{I I} g_{I I}\right) E}}{\left(g_{1}\right)}\right\} .
$$

Knowing $f: R_{A} \rightarrow \hat{R}_{\hat{A}}$ allowable for any one fixed pair of geometrically significant forms $\Lambda, \hat{\Lambda}$ gives dilatation for that pair as a function of principal curvatures at corresponding points. This, using (7), gives the ratio

$$
\frac{\frac{\hat{E}}{\hat{E}}}{\frac{\widehat{G}}{G}}=h\left(k_{1}, k_{2} ; \hat{k}_{1}, \hat{k}_{2}\right)
$$

as a function of principal curvature values. Now, given arbitrary geometrically significant $\Lambda, \hat{\Lambda}$, substitution of (8) on the right hand side of (7) gives the new dilatation itself as a function of principal curvature values. Finally, note that $f$ preserves irremovable umbilics, since it preserves lines of curvature. On the other hand, any geometrically significant $\hat{A}$ ' is proportional to $\left(\hat{I}\right.$ ) at an umbilic ${ }^{\prime} \hat{p}$ on $(\hat{S}$. Thus the value of the dilatation for $f$ at an irremovable umbilic $p$ is fixed over all possible geometrically significant pairs $\Lambda, \hat{\Lambda}$. Since dilatation at $p$ can be determined by the curvature values at $p$ and $f(p)$ for one geometrically significant pair $\Lambda, \hat{\Lambda}$, those values automatically determine the same dilatation for any other geometrically significant pair $\Lambda, \hat{\Lambda}$ at the irremovable umbilic $p$.

COROLLARY. If $f: S \rightarrow \widehat{S}$ preserves lines of curvature while $\mathscr{K}_{f}(\Lambda, \hat{\Lambda}) \equiv$ constant for any one geometrically significant pair $\Lambda, \hat{\Lambda}$, then $f: R_{\Lambda} \rightarrow R_{\hat{\Lambda}}$ is allowable for all geometrically significant $\Lambda, \hat{\Lambda}$. 
This corollary implies, for example, that the reflection of a surface in a sphere is allowable between any pair of geometrically significant structures, since both $R_{I}$ structure and lines of curvature are preserved. Less specifically, where $K \cdot \hat{K} \neq 0$ and $f: S \rightarrow \widehat{S}$ preserves normals, the fact that $\mathscr{K}_{f}(I I I, I \hat{I} I) \equiv 1$ leads to the following result.

THEOREM. Let $f: S \rightarrow \widehat{S}$ preserve lines of curvature and normals. Then (except perhaps where $K \cdot \hat{K}=0) f: R_{1} \rightarrow \hat{R}_{\hat{A}}$ is allowable for any geometrically significant pair $\Lambda, \hat{\Lambda}$. Listed below are the $\mathscr{K}_{f}^{2}(\Lambda, \hat{\Lambda})$ values for such an $f$ relative to various familiar conformal structures.

Note. The values which follow apply only where the structures in question are defined, and need not be valid where $K \cdot \hat{K}=0$.

The following pairs $(\Lambda, \hat{\Lambda})$ are considered: $I, \hat{I} ; I, \hat{\Pi} ; I, \hat{\Pi}^{\prime} ; I, I I I$ or $I, I \hat{I} I ; I, \hat{I}+I \hat{I} I ; I I, \hat{I} ; I I, \hat{\Pi}^{\prime} ; I I, I I I$ or $I I, I \hat{I} I ; I I, I+I \hat{I} I ; I I^{\prime}, \widehat{\Pi}^{\prime}$; $I I^{\prime}, I I I$ or $I I^{\prime}, I \hat{I} I ; I I^{\prime}, \hat{I}+I \hat{I} I ; I I I, \hat{I}+I \hat{I} I$ or $I \hat{I} I, \hat{I}+I \hat{I} I ; I+I I I$, $\hat{I}+I \hat{I} I$. The value of $\mathscr{K}_{f}^{2}(\Lambda, \hat{\Lambda})$ for each pair above is the max of the corresponding term in the list below, and its reciprocal.

$$
\begin{aligned}
& \frac{k_{1}^{2} \hat{k}_{2}^{2}}{\hat{k}_{1}^{2} k_{2}^{2}} ; \quad \frac{k_{1}^{2} \hat{k}_{2}}{\hat{k}_{1} k_{2}^{2}} ; \frac{-k_{1}^{2} \hat{k}_{2}}{\hat{k}_{1} k_{2}^{2}} ; \frac{k_{1}^{2}}{k_{2}^{2}} ; \frac{\left(1+\hat{k}_{1}^{2}\right) k_{1}^{2} \hat{k}_{2}^{2}}{\left(1+\hat{k}_{2}^{2}\right) \hat{k}_{1}^{2} k_{2}^{2}} ; \frac{k_{1} \hat{k}_{2}}{\hat{k}_{1} k_{2}} ; \frac{-k_{1} \hat{k}_{2}}{\hat{k}_{1} k_{2}} ; \\
& \frac{k_{1}}{k_{2}} ; \quad \frac{\left(1+\hat{k}_{1}^{2}\right) k_{1} \hat{k}_{2}^{2}}{\left(1+\hat{k}_{2}^{2}\right) k_{2} \hat{k}_{1}^{2}} ; \frac{k_{1} \hat{k}_{2}}{\hat{k}_{1} k_{2}} ; \frac{-k_{1}}{k_{2}} ; \frac{-\left(1+\hat{k}_{1}^{2}\right) k_{1} \hat{k}_{2}^{2}}{\left(1+\hat{k}_{2}^{2}\right) k_{2} \hat{k}_{1}^{2}} ; \\
& \frac{\left(1+\hat{k}_{1}^{2}\right) \hat{k}_{2}^{2}}{\left(1+\hat{k}_{2}^{2}\right) \hat{k}_{1}^{2}} ; \frac{\left(1+\hat{k}_{1}^{2}\right)\left(1+k_{2}^{2}\right) \hat{k}_{1}^{2} \hat{k}_{2}^{2}}{\left(1+k_{1}^{2}\right)\left(1+\hat{k}_{2}^{2}\right) \hat{k}_{1}^{2} k_{2}^{2}}
\end{aligned}
$$

For the values of $\mathscr{K}_{f}^{2}(I I, \widehat{I}), \mathscr{K}_{f}^{2}\left(I I^{\prime}, \hat{I}\right), \mathscr{K}_{f}^{2}\left(I I^{\prime}, \hat{I I}\right)$, etc., interchange $k_{1}$ with $\hat{k}_{1}$ and $k_{2}$ with $\hat{k}_{2}$ in the expressions for $\mathscr{K}_{f}^{2}(I, \hat{I I}), \mathscr{K}_{f}^{2}\left(I, \hat{I I}^{\prime}\right)$, $\mathscr{\mathscr { L }}_{f}^{2}\left(I I, \hat{I}^{\prime}\right)$, etc.

CoRollarY'. For the standard map (where regular) of $S$ onto a parallel surface, for the spherical image map of $S$ (where $K \neq 0$ ), and, of course, for the identity map of $S$ onto itself, dilatation relative to any pair of geometrically significant $\Lambda, \hat{\Lambda} i$ s a function of the principal curvatures on $S$.

Proof. For the standard map onto a parallel surface $t$ units distant, set

$$
\hat{k}_{1}=\frac{k_{1}}{1-t k_{1}}, \quad \hat{k}_{2}=\frac{k_{2}}{1-t k_{2}}
$$

in the expression for $\mathscr{K}_{f}(\Lambda, \hat{\Lambda})$ in terms of $k_{1}, k_{2}, \hat{k}_{1}, \hat{k}_{2}$. For the 
spherical image map, set

$$
\hat{k}_{1} \equiv \hat{k}_{2} \equiv 1
$$

in the same expression. For the identity map, of course, just substitute

$$
\hat{k}_{1} \equiv k_{1}, \quad \hat{k}_{2} \equiv k_{2} .
$$

Note that by the Corollary', the condition of quasi-conformality on any of the three mappings concerned becomes an inequality on the principal curvatures on $S$. Similarly, requiring that $\mathscr{K}_{f}(\Lambda, \hat{\Lambda})$ be constant for geometrically significant $\Lambda, \hat{\Lambda}$, one gets a Weingarten condition on $S$.

Our theorem thus provides an alternative proof for most results in [3], [4], and [7], and can be used, for example, to establish the corresponding statements involving $R_{I+I I I}$ structure, thus describing Teichmüller mappings $\left(f: R_{I} \rightarrow \hat{R}_{\hat{I}+I \hat{I I}}, f: R_{I I} \rightarrow \hat{R}_{\hat{I}+I \hat{I I}}\right.$, etc.) between surfaces in $E^{3}$ which preserve lines of curvature and normals.

3. An application. Each geometrically significant conformal structure on a surface provides a fresh opportunity for the use of complex analysis in the solution of differential geometric problems. An example seems in order of the sort of geometric argument which can be based upon the use of a nonstandard geometrically significant structure, an allowable mapping, and the computation of a dilatation. We choose the proof of a small fact about the ordinary conformal structure of a very special sort of surface.

Fact. If a complete $S$ is immersed smoothly in $E^{3}$ with $K \leqq 0$ and $H^{2}-K \equiv 1$, then $S$ (i.e., $R_{I}$ ) is conformally the plane or the once punctured plane (and is thus in particular parabolic).

REMARK. Requiring $H^{2}-K \equiv c>0$, amounts to saying that principal curvature values differ by $2 \sqrt{c}$. Our normalization to $c=1$ (solely for convenience) makes this difference equal 2.

Proof. The Codazzi equations force the existence in the neighborhood of any point on $S$ of coordinates $x, y$ in terms of which

$$
\begin{aligned}
I & =e^{k} d x^{2}+e^{-k} d y^{2} \\
I I & =k e^{k} d x^{2}+(k+2) e^{-k} d y^{2},
\end{aligned}
$$

where $k$ and $(k+2)$ are the principal curvature values. Since

$$
K=k(k+2) \leqq 0,
$$


we have

$$
-2 \leqq k \leqq 0
$$

On the other hand, since the local coordinates $x, y$ are determined anywhere on $S$ up to additive constants and/or simultaneous multiplication by 1 or -1 , we recognize

$$
\hat{\Lambda}=d x^{2}+d y^{2}
$$

as a flat metric on all of $S$ which is complete because $I$ is complete while

$$
I<e^{2} \hat{\Lambda}
$$

It follows that the universal covering surface $\Sigma$ of $S$ provided with the complete, flat metric $\hat{\Lambda}$ is isometric to the (finite) plane. But then, using (6),

$$
\mathscr{K}_{i d}(I, \hat{\Lambda})=e^{-l} \leqq e^{2}<\infty,
$$

so that $f: i d: R_{I} \rightarrow R_{\hat{\Lambda}}$ lifts to a quasiconformal homeomorphism of $\Sigma$, making $\Sigma$ provided with the metric $I$ conformally the plane. It follows that $R_{I}$ must be the plane, the once punctured plane or the torus. The last possibility is discarded since no compact $S$ exists in $E^{3}$ with $K \leqq 0$.

\section{BIBLIOGRAPHY}

1. L. Ahlfors, On quasiconformal mappings, Journal D'Anal. Math., III, 1 (1953-4), $1-58$.

2. A. Duschek and W. Mayer, Lehrbuch der Differentialgeometrie, I, B. G. Teubner, Leipzig, 1930.

3. T. Klotz, The geometry of extremal quasiconformal mappings, Michigan Math. J. 9 (1962), 129-136.

4. - Some geometric consequences of conformal structure, Trans. Amer. Math. Soc. (1) 108 (1963), 38-53.

5 . - Some uses of the second conformal structure on strictly conuex surfaces, Proc. Amer. Math. Soc. (5) 14 (1963), 793-799.

6. - Another conformal structure on immersed surfaces of negative curvature, Pacific J. Math. (4) 13 (1963), 1281-1288.

7. - Further geometric consequences of conformal structure, Trans. Amer. Math. Soc. (1) 112 (1964), 67-78.

8. D. Struik, Lectures on Classical Differential Geometry, Addison-Wesley, Cambridge, Mass., 1950.

Received July 18, 1966. This research was conducted at UCLA under NSF grant GP-2567, and at the NYU Courant Institute of Mathematical Sciences under a Ford Foundation grant. Results in the paper were announced at the Conference on Quasiconformal Mappings, Moduli, and Discontinuous Groups at Tulane University, May, 1965. 


\section{PACIFIC JOURNAL OF MATHEMATICS}

\section{EDITORS}

\section{H. SAMELSON}

Stanford University

Stanford, California

J. P. JANS

University of Washington

Seattle, Washington 98105
J. Dugundji

University of Southern California Los Angeles, California 90007

RICHARD ARENS

University of California

Los Angeles, California 90024

\section{ASSOCIATE EDITORS}
E. F. BECKENBACH
B. H. NeumanN
F. WOLF
K. YosidA

\section{SUPPORTING INSTITUTIONS}

UNIVERSITY OF BRITISH COLUMBIA

CALIFORNIA INSTITUTE OF TECHNOLOGY

UNIVERSITY OF CALIFORNIA

MONTANA STATE UNIVERSITY

UNIVERSITY OF NEVADA

NEW MEXICO STATE UNIVERSITY

OREGON STATE UNIVERSITY

UNIVERSITY OF OREGON

OSAKA UNIVERSITY

UNIVERSITY OF SOUTHERN CALIFORNIA
STANFORD UNIVERSITY

UNIVERSITY OF TOKYO

UNIVERSITY OF UTAH

WASHINGTON STATE UNIVERSITY

UNIVERSITY OF WASHINGTON

AMERICAN MATHEMATICAL SOCIETY CHEVRON RESEARCH CORPORATION TRW SYSTEMS

NAVAL ORDNANCE TEST STATION 


\section{Pacific Journal of Mathematics \\ Vol. 22, No. $1 \quad$ January, 1967}

Charles A. Akemann, Some mapping properties of the group algebras of a

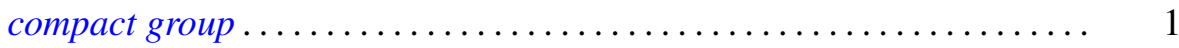

A. V. Boyd, Note on a paper by Uppuluri ..................... 9

Thomas Craig Brown, A semigroup union of disjoint locally finite subsemigroups which is not locally finite .....................

Richard Thomas Bumby and Everett C. Dade, Remark on a problem of

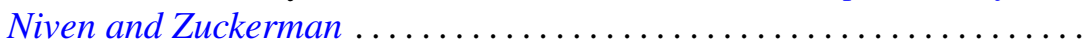

James Calvert, An integral inequality with applications to the Dirichlet problem .........................................

Jack Gary Ceder and Terrance Laverne Pearson, On products of maximally resolvable spaces ....................................

William Guignard Faris, The product formula for semigroups defined by

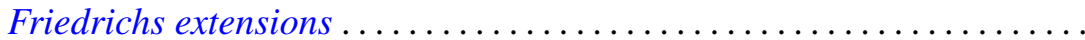

Robert S. Freeman, Closed operators and their adjoints associated with elliptic differential operators ........................ 71

Thomas Lee Hayden, The extension of bilinear functionals ............. 99

Gloria Conyers Hewitt, Limits in certain classes of abstract algebras . . . . . 109

Tilla Weinstein, The dilatation of some standard mappings ........... 117

Mitsuru Nakai, On Evans' kernel ......................... 125

Ernest Levane Roetman, On the biharmonic wave equation ............ 139

Malcolm Jay Sherman, Operators and inner functions ... . .

Walter Laws Smith, On the weak law of large numbers and the generalized elementary renewal theorem

A. J. Ward, On H-equivalence of uniformities: The Isbell-Smith problem 